\title{
Devises, armoiries et portraits d'Aymon de Montfalcon : un évêque en représentation
}

\section{Brigitte Pradervand}

\section{(2) OpenEdition}

\section{Journals}

Édition électronique

URL : http://journals.openedition.org/edl/1454

DOI : $10.4000 /$ edl. 1454

ISSN : 2296-5084

\section{Éditeur}

Université de Lausanne

\section{Édition imprimée}

Date de publication : 1 décembre 2018

Pagination : 311-334

ISBN : 978-2-940331-69-7

ISSN : 0014-2026

\section{Référence électronique}

Brigitte Pradervand, « Devises, armoiries et portraits d'Aymon de Montfalcon : un évêque en représentation », Études de lettres [En ligne], 3-4 | 2018, mis en ligne le 15 décembre 2020, consulté le 17 décembre 2020. URL : http://journals.openedition.org/edl/1454; DOI : https://doi.org/10.4000/edl. 1454

\section{(c) Études de lettres}




\title{
DEVISES, ARMOIRIES ET PORTRAITS D'AYMON DE MONTFALCON : UN ÉVÊQUE EN REPRÉSENTATION
}

\begin{abstract}
Aymon de Montfalcon, mécène, prince et évêque, a laissé dans la région lausannoise un héritage artistique très conséquent. Un des grands intérêts de ce patrimoine réside, comme le montrent les différentes contributions réunies ici, dans la diversité à la fois des œuvres conservées, mais aussi des supports qui ont accueilli ces commandes. Peintres, enlumineurs, écrivains, verriers, sculpteurs, menuisiers, tailleurs de pierre, architectes se sont mis au service de ce prélat hors du commun, créant ainsi un réseau artistique complexe entre le Bugey, la région de France voisine d'où est originaire la famille de l'évêque, la Bourgogne, la Loire et la capitale du pays de Vaud, Lausanne.
\end{abstract}

À la lumière de nouvelles découvertes de peintures murales effectuées dans le cadre de la restauration du château Saint-Maire (voir pl. I et XII), grâce aussi à l'identification d'une image restée jusque là mystérieuse et, enfin, grâce au réseau personnel de l'évêque récemment mis en évidence, des liens peuvent être tissés maintenant entre les œuvres, les artistes et les personnes de l'entourage d'Aymon de Montfalcon. Se mêlent ainsi de manière très étroite les trois aspects de la biographie du prince, de l'évêque et du mécène, l'une des facettes permettant d'éclairer les deux autres.

On connaissait depuis le XIXe siècle au moins la salle du château, nommée "chambre de l'évêque», qui fit l'objet d'une importante rénovation en 1847 déjà $^{1}$ (voir pl. XIII). Les restaurations du monument

I. C. Huguenin, D. Lüthi, Le château Saint-Maire, histoire du Château et de ses restaurations, rapport ms février 2004; M. Grandjean, La ville de Lausanne, p. 364-372 et Lausanne, villages et hameaux de l'ancienne campagne vaudoise, p. 363-365. 
entreprises au début du $\mathrm{XX}^{\mathrm{e}}$ siècle permirent encore la découverte, en 1908 , de très intéressantes peintures ornant les parois du corridor du château. En même temps, on mit au jour dans l'ébrasement des deux fenêtres de la salle dite de conférence des peintures commandées par le prédécesseur d'Aymon de Montfalcon, l'évêque Benoît de Montferrand, que l'on attribua au peintre dijonnais Pierre Spicre ${ }^{2}$.

Dès lors, de nombreux articles évoquèrent les peintures du corridor, mettant toujours l'accent sur leur style renaissant, une des premières manifestations de ce genre en Suisse romande ${ }^{3}$. Si l'attribution du texte de la paroi sud, soit le Bréviaire des nobles d'Alain Chartier, se fit rapidement, en revanche, il fallut attendre les années 1980 pour que les textes de la paroi nord, celui des Douze Dames de Rhétorique, soit clairement identifié $^{4}$. En 1952, l'on retrouva une curieuse figure dans une niche de la salle de conférence. Il s'agit de la représentation de Jeunesse et Fortune. Là encore, l'interprétation de l'image se fit attendre puisque encore tout récemment, on la déclara comme allégorie de la Vérité! ${ }^{5}$ La devise choisie par Aymon de Montfalcon, si qua fata sinant, suscita aussi beaucoup de questions et l'on s'étonna à plusieurs reprises du choix de ce texte profane par un ecclésiastique, la notion de destin paraissant quelque peu incongrue à nos prédécesseurs ${ }^{6}$. Enfin, le fameux monogramme formé des lettres $\mathrm{A}$ et $\mathrm{M}$ entrelacées qui se voit en différents lieux fit également couler beaucoup d'encre, sans que son interprétation fasse l'unanimité dans la communauté des historiens.

Que révèle cette difficulté d'interprétation des œuvres commandées par l'évêque? Pourquoi leur compréhension a-t-elle résisté à de

2. Sur ces peintures, voir notamment: $M$. Reymond, "Un peintre dijonnais à Lausannne au XVe siècle"; A. Decollogny, "Peintures murales au château SaintMaire, chapelle Saint-Nicolas, Lausanne"; M. Grandjean, La ville de Lausanne; E. Castelnuovo, Th.-A. Hermanès, «La peinture du Moyen Âge», p. 70 ; F. Joubert, "Nouvelles propositions sur la personnalité artistique de Pierre Spicre»; B. Pradervand, "Les peintures murales du château Saint-Maire», p. 68-83.

3. J.-A. Bohy, «Les fresques pré-renaissantes du château de Lausanne» et «Les peintures du château de Lausanne"; E. Castelnuovo, Th.-A. Hermanès, "La peinture du Moyen Âge», p. 70 ; F. Elsig, «La peinture en Savoie et en Franche-Comté durant la première moitié du XVI ${ }^{\mathrm{e}}$ siècle», p. 85.

4. M.-R. Jung, "Les "Douze Dames de rhétorique” "; J.-C. Mühlethaler, "Ein dichterisches und politisches Manifest in Lausanne» et "Satire et recyclage littéraire».

5. Cf. l'article de J.-C. Mühlethaler dans ce volume et en particulier la fig. 2.

6. M. Reymond, «Si qua fata sinant». 
nombreuses analyses? Quelles places occupent ces multiples signes identitaires, laissés à dessein? Quels liens entretiennent-ils avec les œuvres elles-mêmes et, in fine, quel sens leur donner? C'est ce que nous allons tenter de décrypter.

\section{Indices biographiques}

Si les grands prélats de ce temps n'ont pas manqué de marquer leurs commandes artistiques de leurs armoiries, de leur devise, voire n'ont pas hésité à se faire représenter au sein même de l'œuvre, peu ont toutefois laissé autant d'occurrences. Ces marques personnelles laissées par Aymon de Montfalcon remontent pratiquement toutes aux années 1500 et postérieures. Il est alors âgé d'environ 60 ans. Cet homme d'une grande culture, nourri des ouvres antiques, mais aussi de celles de ses contemporains, s'entoura, pendant les quelques années de son épiscopat, des meilleurs artistes pour livrer à la postérité des œuvres que nous admirons encore aujourd'hui.

Aymon de Montfalcon en eut largement conscience, on le sait notamment par une longue inscription conservée dans son village de Flaxieu. Il y avait édifié l'église, vers 1483, avec son frère Hugonin, et il y construisit également une fontaine monumentale, surmontée d'un cartouche dans lequel ses qualités sont décrites. Le texte lui attribue des dons reçus de la muse Calliope, ainsi qu'en témoigne l'extrait suivant, traduit du latin:

Aymon, au sang illustre, bâtit ce monument

Pour l'étranger; vraiment c'est œuvre de noblesse.

Montfalcon a du talent, les Lycurgue lui ont accordé les droits Et Calliope ses titres ${ }^{7}$

La manière d'inscrire dans la matière ces indices biographiques, dans des contextes et situations très divers, à la fois religieux, familiaux et profanes, témoigne de cette volonté récurrente de personnaliser ses commandes. Mais en même temps qu'il signe en quelque sorte les ouvrages produits, il crée des énigmes et la difficulté de leur

7. Cité par M. Grandjean, L'architecture religieuse en Suisse romande et dans l'ancien diocèse de Genève à la fin de l'époque gothique, p. 706; L. de Montfalcon, "Aymon de Montfalcon, prélat de la Renaissance», 1969, p. 187. 
identification, même de la part de chercheurs chevronnés, révèle bien la complexité de sa démarche artistique. Nous sommes face à des rébus, des jeux mettant en scène des emblèmes, quelques années avant les célèbres publications des emblèmes d'Alciat ou plus tard de Cesare Ripa. Toutes ses œuvres entrent en résonnance étroite avec son vécu, mais de manière masquée pour les non-initiés, telle la situation d'un narrateur dans une posture littéraire. Cette distance, sorte de jeu de pistes avec le spectateur, devait également paraître comme telle à ses propres contemporains, comme nous allons le voir.

La découverte récente d'une salle peinte, oubliée dans le château Saint-Maire depuis 1952, permet de mettre en perspective toutes ces occurrences.

\section{La mise en scène des armoiries familiales}

En même temps que l'allégorie de Fortune et de Jeunesse découverte dans la niche ménagée dans la paroi nord de la salle de conférence, le restaurateur d'art Ernest Correvon mit au jour, en 1952, des peintures qui furent ensuite rapidement dissimulées. Elles viennent de réapparaître sous une tenture ${ }^{8}$.

Trois parois sont ornées de décors peints, certes conservés de manière fragmentaire, mais d'un grand intérêt tant du point de vue historique qu'artistique. Trois registres distincts animent la hauteur du mur: un soubassement, dont on notera la proximité formelle avec celui du corridor, une partie médiane agrémentée de fines colonnettes et d'éléments végétaux et, enfin, une frise armoriée. Cette dernière comprend de petites figures d'une grande qualité picturale, œuvre d'un artiste pour l'heure inconnu' (voir pl. XIV). Les peintures sont assurément de la même main que celles du corridor, mais contrairement à ces dernières qui ont subi une forte restauration au début du $\mathrm{XX}^{\mathrm{e}}$ siècle, elles n'ont souffert d'aucune intervention depuis leur origine.

8. Quelques photographies témoignent de la découverte, mais on ignore pour quelle raison les peintures furent ensuite cachées sous une tenture. Ces décors sont en cours de restauration par l'Atelier Saint-Dismas et feront prochainement l'objet d'une publication.

9. Voir à ce propos: B. Pradervand, "Les peintures murales du château SaintMaire», p. 68-83. 
Fig. 1 - Armoiries sur la façade du corps de logis du château de Glérolles.

Photographie Claude Bornand, 1972.

Les écus représentés sont tous partis avec, à gauche, les armoiries de la famille Montfalcon "écartelé aux 1 et 4 d'argent au faucon éployé de sable, armé d'or aux 2 et 3 contre-écartelé d'hermine et de gueules» et à droite, les diverses alliances des frères de l'évêque Aymon, notamment de son frère aîné Hugonin (Menthon), de François (Rochette) et peut-être de Louis ${ }^{10}$. Les armoiries des parents d'Aymon, de son père Guillaume de Montfalcon et de sa mère Marguerite de Chevron-Villette, figurent en tête de la série.

Pourquoi cette salle armoriée? Et pourquoi sur trois parois seulement? Les peintures commandées par Aymon de Montfalcon tenaient visiblement compte de celles laissées par son prédécesseur, Benoît de Montferrand, sur la quatrième paroi, au nord. Ces associations héraldiques ne sont pas un unicum dans les œuvres commandées par l'évêque.

Io. D. L. Galbreath, Armorial vaudois; É.-A. de Foras, Armorial et nobiliaire de l'ancien duché de Savoie. 
Fig. 2 - Vue générale des stalles de la chapelle d'Aymon de Montfalcon à la cathédrale de Lausanne. Photographie Jeremy Bierer, 2018.

Un autre exemple se trouve au château de Glérolles, au bord du lac Léman, propriété de l'évêché dès 1303. Les mêmes séquences d'armoiries apparaissent sur une façade du corps de logis transformée précisément par Aymon de Montfalcon. Elles sont, dans ce cas, sculptées dans des médaillons de pierre (fig. 1). Cette parenté entre les deux résidences s'observe encore avec la proximité formelle de cheminées au décor pratiquement identique ${ }^{11}$. Ce sont toutes deux des propriétés de l'évêché certes, mais mises au service du lignage du prince, afin de mettre en exergue son réseau familial.

Sur les stalles de sa chapelle (fig. 2), dans la cathédrale de Lausanne, Aymon de Montfalcon a également disposé les armoiries familiales, celles de son père et de sa mère dont les tenants sont des licornes ou des bouquetins. Deux lettres, $\mathrm{G}$ et $\mathrm{M}$, identifient les prénoms de ses parents, Guillaume et Marguerite. Il a également fait sculpter ses propres armoiries couronnées de la mitre, accompagnées de la crosse et de la palme, disposées dans des endroits bien précis, où il est représenté en dévotion

II. Voir fig. 7-9 de la contribution de M. Grandjean dans ce volume, ainsi que la planche XIII. Cf. également M. Grandjean, L'architecture religieuse en Suisse romande et dans l'ancien diocèse de Genève à la fin de l'époque gothique, p. 574-579. 
(pl. XVI). Ailleurs, ce sont les armoiries du prince, du seigneur temporel qui sont sculptées ${ }^{12}$.

Ce sont aussi certainement des liens familiaux qui expliquent la présence du texte des Douze Dames de Rhétorique à Lausanne. Jean de Montferrant(-d), l'un des protagonistes du récit, est le frère de l'évêque Benoît de Monferrand, le prédécesseur de Montfalcon. Mais il est aussi le frère de Claude de Montferrand qui est, depuis 1474, l'époux d'Alix, sœur d'Aymon. Et voilà pourquoi Aymon a conservé les décors de son prédécesseur dans cette salle de conférence dédiée à son lignage, des peintures d'un style très différent de ce qu'il devait apprécier et qui devaient peut-être paraître désuètes vers $1500^{13}$. Il est même plausible de se demander si le beau-frère d'Aymon, Claude de Montferrand, voire sa sœur, Alix, ne figurent pas sur un des portraits formant la suite de l'évêque Benoît de Montferrand.

Mais Aymon de Montfalcon ne s'est pas contenté d'inscrire sur les murs des témoignages de son lignage. Il a également mis en scène très soigneusement le contenu du propos de l'œuvre. La disposition des figures dans le corridor du château Saint-Maire est particulièrement intéressante et révèle la culture du prélat (voir pl. IV).

Tout d'abord, c'est bien sur un mur que l'évêque va faire écrire ce texte, et non sur un support habituel pour un si long discours, livre ou tapisserie. Ce n'est a priori pas très commode, ni pour le peintre, ni pour le lecteur. Et ensuite, il va encore le mettre en résonnance et en dialogue avec un autre texte: le Bréviaire des nobles. Ce dernier exprime les vertus que doit avoir un homme de noble condition: Noblesse, Loyauté, Honneur, Droiture, Prouesse, Diligence et Netteté. Comme l'a déjà mis en évidence Jean-Claude Mühlethaler, cette commande ne constitue pas une simple superposition d'œuvres, qui pourrait être de type purement esthétique, mais les textes entrent bien aussi en résonnance

I2. E. Bach, L. Blondel, A. Bovy, La cathédrale de Lausanne; E. Dupraz, "La chapelle des Thébéens dans la cathédrale de Lausanne»; E. Bach, "Les stalles gothiques de Lausanne»; C. Lapaire, "Les stalles de la chapelle des Martyrs thébains"; B. Pradervand, N. Schätti, «Les stalles», p. 184-189; C. Lapaire, «Les stalles de la cathédrale de Lausanne».

I3. C'est ce que révèlent les images prises lors de la découverte des peintures. Sous le badigeon, commun à toute la salle, apparaissent directement ces peintures, sans les motifs présents sur les trois autres parois de l'époque de Montfalcon. 
sémantique à la fois littéraire et politique ${ }^{14}$. Deux livres sur les murs! Ce n'est pas commun! Les pages se tournent en cheminant dans le long couloir qui donne accès aux salles principales du château. De plus, Les Douze Dames de Rhétorique est un livre savant, dont l'identification prit un certain temps depuis sa découverte, preuve de son caractère exceptionnel, et dont les contemporains d'Aymon ne devaient, pour la plupart, pas y comprendre grand-chose. Le style des peintures ne correspond à aucun des trois manuscrits enluminés conservés des Douze Dames de Rhétorique attribués au maître d'Antoine de Bourgogne ${ }^{15}$. Il ne s'agit donc pas de simples copies d'œuvres antérieures, mais bien d'une nouvelle approche artistique, thématique, sémantique, et même politique de la part d'Aymon. Que comprendre derrière cette volonté? Aymon de Montfalcon a-t-il voulu impressionner le visiteur en attente d'une audience? A-t-il voulu témoigner de sa culture? Introduire des idées nouvelles? Peut-être bien tout cela à la fois.

\section{Si qua fata sinant}

Cette appropriation des textes littéraires contemporains s'accompagne d'autres éléments très personnels. La devise si qua fata sinant est inscrite à de multiples reprises dans le corridor et se joint au propos (voir pl. VIII.2).

Elle surmonte les figures féminines du château Saint-Maire et soustend une frise formée d'écus dont les supports et tenants appartiennent clairement au répertoire renaissant (centaures, putti, etc.). Cette devise personnelle est extraite du premier chant de l'Énéide (Æn. I, 18) et peut être traduite par «si le (ou les) destin(s) le permet(tent)». Dans le texte de Virgile, la déesse Junon a le projet de faire régner sur le monde la ville de Carthage «si le destin le veut». Elle demande à Éole de déclencher une tempête, afin de détruire les bateaux des Troyens arrivés proches des côtes de l'Italie. Elle sait pourtant que le destin le lui interdit ... Son projet échouera, Énée pourra accoster avec l'aide de Neptune et la

I4. J.-C. Mühlethaler, «Ein dichterisches und politisches Manifest in Lausanne» et "Satire et recyclage littéraire».

15. K. Straub, Les Douze Dames de Rhétorique, Studien zu einer französischen Handschrift des 15. Jahrhunderts, p. 150 sq. 
ville de Rome sera fondée par les descendants des Troyens. Si l'on tient compte de ce contexte, le destin est donc une notion très ambivalente. Le fait que cette citation intervienne tout au début de l'Énéide confirme, si l'on pouvait en douter, qu'Aymon de Montfalcon connaît évidemment l'origine de sa citation ${ }^{16}$.

Presque partout où Aymon de Montfalcon est intervenu, il a fait graver ses armoiries et sa devise par ses tailleurs de pierre, ses ébénistes, ses verriers ou ses tisserands. Et toujours de belle manière, les associant au décor flamboyant ou renaissant, avec des lettres sculptées ou peintes dans de très beaux caractères. Avec de grandes variations de grandeur, de styles, insérant parfois la date au centre de l'œuvre, comme à la tribune de la cathédrale. Il serait trop long de citer ici toutes les occurrences, que ce soit sur les monuments tels que le château de Glérolles, la cathédrale de Lausanne, l'église et le château de Curtilles ${ }^{17}$, ou sur du mobilier ${ }^{18}$, ou divers objets personnels. Parfois, la devise est si présente qu'elle est complètement apparentée à l'œuvre elle-même jusqu'à faire partie intégrante du décor, voire à le créer, la forme finissant par se libérer du sens comme à l'entrée de la chapelle de la cathédrale (fig. 3). Sur les ornements sacerdotaux qu'il a commandés, la devise figure audessus de chaque scène ornant la chasuble de l'évêque et les dalmatiques des diacres. La présence d'une devise sur des vêtements liturgiques n'est pas exceptionnelle, mais une telle répétition paraît rare selon Annemarie Stauffer qui a étudié ces objets ${ }^{19}$.

On aurait tort de penser que la présence ou non de la devise est le fruit du hasard. Il y a une distinction dans son usage selon le contexte. Par exemple, dans le cas du bréviaire de Lausanne, les armes d'Aymon, accompagnées de celles du chapitre, ne comportent pas la devise ${ }^{20}$. Le commanditaire a choisi soigneusement les endroits dans lesquels elle devait figurer. Il a donné des consignes précises à tous ses maîtres d'œuvre.

I6. Sur le rapport d'Aymon de Montfalcon avec l'Antiquité, cf. l'article de G. Oguey dans ce volume et M. Grandjean, "Jalons pour une histoire de la conservation des monuments historiques vaudois jusqu'à Viollet-le-Duc», p. 73; et sur le thème de la Fortune: J.-C. Mühlethaler, «Satire et recyclage littéraire» et «Quand Fortune, ce sont les hommes", p. 194-204.

I7. M. Fontannaz, B. Pradervand, Le district de la Broye-Vully I, p. 278-294.

I8. Cathédrale de Lausanne. $700^{e}$ anniversaire de la consécration solennelle, p. 88.

19. A. Stauffer, D'or et de soie ou les voies du salut.

20. Cathédrale de Lausanne. $700^{e}$ anniversaire de la consécration solennelle, p. 160. 
Fig. 3 - Devise d'Aymon de Montfalcon dans sa chapelle à la cathédrale de Lausanne. Photographie Brigitte Pradervand, 2017.

Comme nous l'avons déjà mentionné, les armoiries représentées dans la salle de conférence sont associées à la figure de la Fortune. Par contre, il est intéressant de noter, dans la perspective d'une filiation des images et des contenus, la parenté de cette figure avec celle ornant un recueil de dessins réalisé par ou pour François Robertet, secrétaire de la duchesse de Bourbon, fils du poète Jean Robertet, l'un des auteurs des Douze Dames de Rhétorique. Il faut donc souligner encore une fois les liens entre les personnes que connaît Aymon et le réseau artistique que l'on commence à mettre en évidence. Si cette image du recueil est bien la source iconographique de la peinture du château, Aymon de Montfalcon ne s'est pas contenté de la reprendre telle quelle, mais il y a apporté sa touche personnelle. Il a modifié le quatrain qui surmonte la peinture, dans le sens d'une certaine empathie, comme le montre Jean-Claude 
Mühlethaler, mais aussi la figure elle-même qui, au lieu de regarder de face son destin se briser contre le roc de Fortune, tourne la tête en arrière en direction des représentations familiales peintes dans la salle ou vers le passé? En conjuguant le sens de cette représentation avec celui de la devise, et avec les armoiries familiales, des éléments biographiques de l'évêque apparaissent plus distinctement, la Fortune ayant joué un rôle important dans la vie d'Aymon selon le témoignage de ses propres écrits.

\section{Le monogramme $A M / M A$}

Avant d'évaluer ces traits biographiques, il faut évoquer cet autre élément équivoque utilisé par Aymon de Montfalcon. Parfois, l'évêque a associé à sa devise les lettres A et M entrelacées (voir pl. XV.1). Sur le plafond à caissons de sa chambre dans le château Saint-Maire, le monogramme est infiniment répété, dans des couleurs différentes, peint en alternance avec des fleurs, la devise cernant l'ensemble de la composition. Sur la stalle d'honneur de la chapelle de la cathédrale de Lausanne, où six faucons constituent les armoiries parlantes des Montfalcon, tout en haut du siège d'honneur, sur les dais, disparaissant dans le fourmillement de la sculpture, se trouvent les lettres A et M. Les deux lettres, à l'église de Curtilles, sont peintes sur un vitrail. Elles étaient peut-être également figurées dans un manuscrit qui lui appartenait contenant des extraits du Roman de la Rose et des ouvres d'Alain Chartier ${ }^{21}$. Ces deux lettres sont partout entrelacées de la même manière, les jambages commençant d'abord par le $\mathrm{M}$, ensuite le $\mathrm{A}$, puis le $\mathrm{M}$ et le $\mathrm{A}$. Il est donc délicat de décider s'il s'agit de AM ou de MA! Ces deux lettres font bien sûr référence à ses prénom et nom, comme à la salutation angélique Ave Maria, et au monogramme de Marie MA, mais pas seulement. Si la devise accompagne une grande partie des commandes de l'évêque, les lettres A et $\mathrm{M}$ en revanche n'y figurent pas toujours. Par exemple, elles ne se trouvent ni sur les parties transformées de la cathédrale à l'usage du chapitre, ni sur les ornements sacerdotaux destinés eux aussi à la cathédrale et conservés à Berne, mais bien sur le siège d'Aymon de Montfalcon. Ce monogramme est généralement associé, comme c'est le

2I. A. Piaget, "Aymon de Montfaucon et sa cour littéraire», p. 177. Ce manuscrit serait à réexaminer pour confirmer son appartenance. 
cas dans la chambre du château, plutôt à un usage de type privé. Sur les vitraux de l'église de Curtilles, dont le chœur fut réaménagé par Aymon de Montfalcon, ces décors n'étaient pratiquement pas visibles depuis la nef, mais disposés sur le vitrail d'une fenêtre latérale.

Arthur Piaget a, le premier, proposé de voir dans ce monogramme une relation avec un texte composé par l'évêque. Cette idée fut reprise par Louis de Montfalcon ${ }^{22}$. Si la pertinence de cette interprétation romantique pouvait être mise en doute sur la base de la seule présence de ce monogramme, les nouvelles découvertes picturales et leur interprétation, ainsi que la mise en évidence visuelle de l'importance du réseau familial et du lignage de l'évêque, donnent une nouvelle force à cette proposition. Cette approche se trouve confortée aussi par l'examen de deux autres textes, l'un de la main même d'Aymon et un autre texte rédigé par maître Antitus Favre, son chapelain.

À la bibliothèque de Turin se trouvait en effet un manuscrit intitulé Le procez du banny à jamais du Jardin d'Amours. Il disparut malheureusement dans l'incendie de 1904, mais nous le connaissons grâce à la copie partielle qu'en firent deux érudits ${ }^{23}$. Le narrateur, qui se prénomme Ayme, se décrit chevauchant dans la campagne. Il pense à son rêve de la nuit précédente: il assistait à un tournoi lorsqu'il vit une dame, très belle, dont il s'éprit immédiatement. En pensée dans le «Verger d'Amour", il aperçoit un oiseau dans un laurier qui tient un rouleau sur lequel est écrit: "Va-t'en, ton prince te demande». S'ensuit un combat entre Plaisir et Raison. Cette dernière décide que le jeune Ayme doit retourner à Flaxieu et quitter ce lieu de délices. Le récit remonte probablement aux années 1473 . Aymon a trente ans et il vient d'être nommé ambassadeur du roi de Chypre à Rome. Le récit continue par une parole de sa dame qui, très affligée par le départ de son aimé, prend une lettre de son nom et lui demande:

[...] mectez la en vos armes, Mon ami, je vous en fait don

Le narrateur poursuit:

Et pour monstrer amour entiers, une des miennes ay eraschee

22. L. de Montfalcon, «Aymon de Montfalcon, prélat de la Renaissance».

23. Arthur Piaget et Egidio Gorra, cf. A. Piaget, "Aymon de Montfaucon et sa cour littéraire», p. 171. 
laquelle est, par subtilz ouvrier avec la sienne entrelassée.

En aiguières, en pos, en plas

Bassins, tapis et couverture,

Et d'autre choses a grant tas

L'ay fait bouter en pourtraicture

En aucuns lieu tout en painture

Composé de vives couleurs.

En aultres ils sont en brodeure

De fins or et de pierre plusieurs ${ }^{24}$

Le texte ne mentionne pas les lettres en question, mais à la fin du poème se voyaient les fameuses deux lettres $\mathrm{A}$ et $\mathrm{M}$ entrelacées... Dans un autre texte attribué à Aymon de Montfalcon, qui se nomme Le Débat du gris et $d u$ noir, deux personnages dialoguent ${ }^{25}$. Le Gris, homme solitaire, aimerait profiter du monde et de l'amour. Mais il ne le peut. Il vient chaque jour dans un verger merveilleux où il rencontre un passant, le Noir. Ce dernier l'entend murmurer le nom de sa dame que pourtant le poète ne voulait pas nommer en public (!). Il s'arrête et lui donne le conseil suivant:
Aussy, ami, je te conseille
que tu escripves en mains lieux
une devise non pareille,
ou, dessus, mettras pour le mieulx
Deux lettres du nom précieux
De ta deesse terrienne,
par lesquelles faux envieux
Ne sauront la volonté tienne ${ }^{26}$
Celui qui porte $\mathrm{M}$ et $\mathrm{A}$
en plusieurs lieux pour sa devise
Pour celle que tant amee a
Et ayme si qu'ailleurs ne vise
Requierts, se nul facteur avise

24. D’après A. Piaget, «Aymon de Montfaucon et sa cour littéraire», p. 176 sq.

25. Deux manuscrits sont conservés, un à la Bibliothèque nationale de France: Dialogue entre le Gris et le Noir, en vers, BnF 25 421, l'autre dans la bibliothèque de feu le baron James de Rotschild.

26. D’après A. Piaget, "Aymon de Montfaucon et sa cour littéraire», p. 182. 
Aucune faute en ce traitié

Ainsy que de rime on devise

Qu'il n'en soit ja trop mal traittié27

Ce texte fait donc référence à deux éléments distincts: d'une part la devise elle-même, dite "non pareille» et d'autre part deux lettres de la «déesse terrienne» du narrateur, dont le nom doit être tu, à mettre audessus de la devise.

C'est exactement ce qu'Aymon a fait. Et même jusque dans ses stalles, les lettres se perdent dans le décor des dais, mais sont bien présentes au-dessus de son portrait. Son appel à de hautes charges dès 1471 , puis son départ pour Rome en 1473 en ont bien fait un homme d'Église condamné à renoncer à ses amours ${ }^{28}$. Si l'on pouvait encore douter de l'implication directe de la vie d'Aymon dans ses œuvres, il faut encore relire le Portail du temple Bocace, œuvre d'Antitus Favre, écrite dans la lignée du Temple de Bocace de Georges Chastelain, l'un des auteurs des textes peints dans le corridor du château Saint-Maire ${ }^{29}$. Le thème est celui de personnages célèbres qui ont connu un destin tragique. Aux sources du récit, dans les premières pages, est relatée l'histoire dramatique de la mort du neveu d'Aymon, Louis de Montfalcon, fils de Louis, frère de l'évêque. Selon le récit d'Antitus, le petit Louis, âgé de six ans, rendait visite à ses amis, à son souverain et à son oncle, lorsqu'il s'est fait mordre par un chien enragé. Antitus donne la parole à l'enfant qui raconte son malheur et le chagrin immense de son père, de sa mère et de son oncle, l'évêque, en rappelant en passant l'importance de son lignage:

Helas mon oncle bien avez cueur piteux

Jay ung myen oncle evesque de renom prince puissant faisant forger monnoye Jay noble puissant et renomme baron En mon lignage on le voit en savoye ${ }^{30}$

27. D’après A. Piaget, "Aymon de Montfaucon et sa cour littéraire», p. 183.

28. Helvetia Sacra, I/4, p. 146-148.

29. Manuscrit conservé aux Archives cantonales vaudoises (ACV), P. Antitus, consulté en ligne sur e-codices <https://www.e-codices.unifr.ch/fr/list/one/acv/PAntitus>.

30. ACV, P. Antitus, fol. 29v. 
L'enfant demande que l'on couche son corps en un portail, près de quelques piliers. Le fameux temple était fait d'étrange façon:

luysant dedens, le dehors admirable

et en estoit fortune le masson

pave de deuil et les cloches dung son

si esgare quil nest cil qui ne tramble

$[\ldots]^{31}$

Auprès du temple se trouve un cimetière. Louis apparaît dans ce texte comme le fondateur de ce portail. Nous sommes en l'an 1501 et le maçon se nomme... «Fortune».

On ne peut s'empêcher une fois encore de faire le lien avec l'œuvre future du portail de la cathédrale de Lausanne, même si, évidemment il s'agit ici d'une figure littéraire et d'un portail symbolique ${ }^{32}$. Le texte devrait être analysé en détail. Mais comme dans les autres récits, une fois encore le réel fait irruption dans le texte commandé par notre évêque, brouillant les pistes.

\section{Les portraits}

Avant de conclure, il faut encore évoquer les quelques portraits que nous conservons de l'évêque. Il est représenté à trois reprises dans le livre d'Antitus Favre évoqué plus haut, dans un cadre profane (une salle du château Saint-Maire?), sans les attributs qui pourraient l'identifier comme évêque (fig. 4). Aymon de Montfalcon est assis sur un siège surélevé pourvu d'un dais recouvert d'un tissu vert uniforme et, à l'intérieur, d'une tenture rouge. De ces images se dégage une sorte de sobriété, qui ne reflète pas la puissance d'un seigneur spirituel ou temporel, mais s'apparente plus aux écrivains de son temps, voire aux portraits de Luther ou d'Érasme. Seuls signes de richesse: des bordures de fourrure émergent du col et des manches et de légers ornements apparaissent sur la tenture rouge. Le décor de la salle rappelle les peintures de la salle de conférence.

3I. ACV, P. Antitus, fol. 26.

32. Sur les tribulations de l'évêque et la construction du portail dit de «Montfalcon» de la cathédrale, voir l'article de K. Queijo dans ce volume. 
Fig. 4 - Aymon de Montfalcon. Détail du fol. 24r du manuscrit d'Antitus (Archives cantonales vaudoises) (<https://www.e-codices.ch/fr/list/one/acv/P-Antitus >). 


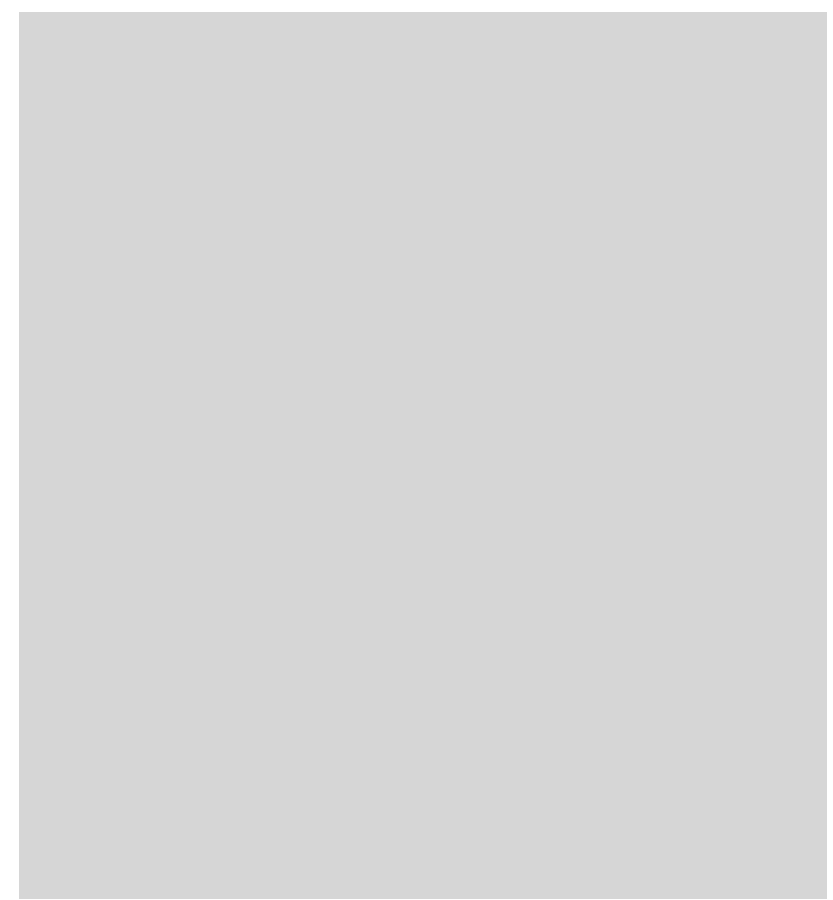

Fig. 5 - Portrait d'Aymon de Montfalcon sur un vitrail de l'église de Curtilles. (C) ACV, Archives des monuments historiques, A 51/3.

Plus curieux encore est le portrait conservé dans le chœur de l'église de Curtilles (fig. 5), possession des évêques de Lausanne. Si les autres vitraux ne comportaient pas les fameuses lettres AM, les armoiries ainsi que la devise d'Aymon, rien ne permettrait de l'identifier. Le prélat n'est pas représenté dans sa fonction, ce pourrait être un portrait de n'importe quel seigneur temporel, si ce n'est peut-être l'aumusse de fourrure que l'on devine sur ses épaules.

Plus traditionnelles sont les représentations sculptées sur les stalles de sa chapelle dans la cathédrale où il apparaît à deux reprises (fig. 6-7). Sur l'une d'elles, Aymon de Montfalcon est agenouillé, en habit de chœur, accompagné de ses armoiries, au pied des martyrs thébains, sur une autre en prière devant la Vierge à l'Enfant. Quelques traits récurrents semblent caractériser le personnage: un menton bien marqué à fossette, des lèvres plutôt charnues et des traits réguliers, peut-être une certaine douceur volontairement affichée... Quant au portrait figuré sur le ducat d'or, il est difficile de faire le lien avec les autres (voir pl. XV.2). 
Fig. 6 - Portrait de l'évêque sur les stalles de sa chapelle à la cathédrale de Lausanne. Photographie Jeremy Bierer, 2018. 
Fig. 7 - Portrait de l'évêque sur les stalles de sa chapelle à la cathédrale de Lausanne. Photographie Jeremy Bierer, 2018. 
En conclusion, les récentes découvertes de peintures murales, l'identification nouvelle des œuvres et l'analyse serrée des liens familiaux d'Aymon de Montfalcon et de son prédécesseur Benoît de Montferrand permettent de mettre en corrélation les différentes facettes de l'évêque et justifient pleinement que l'on continue les recherches entre nos différentes disciplines. Les allusions biographiques, mises en scène de manière systématique, mais sous forme d'indices, parfois peu explicites, constituent un vrai jeu de piste, tant dans les œuvres littéraires que peintes ou sculptées.

Cette attitude pose la question de l'ambiguïté de la posture. Évêque ou prince ou mécène? En fait les trois à la fois et souvent les trois en même temps. Les évènements de sa vie se révèlent ainsi grâce notamment à l'œuvre de Virgile, d'Antitus Favre ou par l'intermédiaire des peintres, sculpteurs, artistes qui devaient former une cour brillante, exceptionnelle même, à la tête de laquelle cet homme, venu à Lausanne à 50 ans, a donné une résonnance internationale. Les diverses interventions présentées dans ce volume ont permis de tirer un petit fil de l'écheveau pour comprendre ce milieu et ce réseau très dense qui a laissé une partie de ces fils à Lausanne, à la veille de la Réforme, et en plein bouleversement de la Renaissance.

Ce fourmillement artistique et cette agitation fit d'ailleurs écrire à Antitus Favre, son chapelain et écrivain, de manière quelque peu désabusée et ô combien moderne à nos yeux que:

le monde nest qung parc a folz [...]

qui ressemble une fourmiere ou jamais il ny a repos ${ }^{33}$.

Brigitte Pradervand

33. ACV, P. Antitus, fol. 23v. 


\section{BIBLIOGRAPHIE}

BACH, Eugène, "Les stalles gothiques de Lausanne", Indicateur d'antiquités suisses, 31/3 (1929), p. 191-206.

Bach, Eugène, Blondel, Louis, Bovy, Adrien, La cathédrale de Lausanne, Bâle, Birkhäuser, 1944 (Les Monuments d'art et d'histoire du canton de Vaud, 2).

Вону, Juliette-Alice, "Les fresques pré-renaissantes du château de Lausanne», Indicateur d'antiquités suisses, 40/2 (1938), p. 129-141.

—, "Les peintures du château de Lausanne. Première œuvre renaissante en Suisse", Revue historique vaudoise, 47/2 (1939), p. 57-80.

Castelnuovo, Enrico, Hermanès, Théo-Antoine, "La peinture du Moyen Âge", in Les Arts. I. Architecture, peinture, littérature, musique, Lausanne, Éd. 24 Heures, 1976, p. 517-554 (Encyclopédie illustrée du Pays de Vaud, 6).

Cathédrale de Lausanne. $700^{e}$ anniversaire de la consécration solennelle, catalogue d'exposition, Lausanne, Musée historique de l'AncienÉvêché, 1975.

Decollogny, Adolphe, «Peintures murales au château Saint-Maire, chapelle Saint-Nicolas, Lausanne", Revue historique vaudoise, 68 (1960), p. 1-4.

Dupraz, Emmanuel, «La chapelle des Thébéens dans la cathédrale de Lausanne", Revue historique vaudoise, 17 (1909), p. 135-147.

ELsig, Frédéric, «La peinture en Savoie et en Franche-Comté durant la première moitié du XVI $\mathrm{X}^{\mathrm{e}}$ siècle", in La Renaissance en Savoie. Les arts au temps du duc Charles II (1504-1553), éd. par Mauro Natale, Frédéric Elsig, Genève, Musée d'Art et d'Histoire, 2002, p. 77-94. Fontannaz, Monique, Pradervand, Brigitte, Le district de la BroyeVully I, Berne, Société d'histoire de l'art en Suisse, 2015 (Les Monuments d'art et d'histoire du canton de Vaud, 8).

Foras, Élie-Amédée de, Armorial et nobiliaire de l'ancien duché de Savoie, vol. 4, Grenoble, Joseph Allier Éditeur, 1900.

Galbreath, Donald Lindsay, Armorial vaudois, 2 vols, Baugy-surClarens, 1934-1936. 
Grandjean, Marcel, La ville de Lausanne. Introduction, extension urbaine, ponts, fontaines, édifices religieux (sans la cathédrale), hospitaliers, édifices publics, Bâle, Birkhäuser, 1965 (Les Monuments d'art et d'histoire du canton de Vaud, 1).

—, "Jalons pour une histoire de la conservation des monuments historiques vaudois jusqu’à Viollet-le-Duc», Revue historique vandoise, 87 (1979), p. 71-97.

-, Lausanne. Villages, hameaux et maisons de l'ancienne campagne lausannoise, Bâle, Birkhaüser, 1981 (Les Monuments d'art et d'histoire du canton de Vaud, 4).

-, L'architecture religieuse en Suisse romande et dans l'ancien diocèse de Genève à la fin de l'époque gothique, Lausanne, Cahiers d'archéologie romande, 2015 (Cahiers d'archéologie romande, 157-158).

Helvetia sacra, I/4. Le diocèse de Lausanne (VI eiècle-1821), de Lausanne et Genève (1821-1925), et de Lausanne, Genève et Fribourg (depuis 1925), par un groupe d'auteurs, rédaction Patrick Braun, Bâle/ Francfort-sur-le-Main, Helbing \& Lichtenhahn, 1988.

Joubert, Françoise, «Nouvelles propositions sur la personnalité artistique de Pierre Spicre», in La splendeur des Rolin, dir. par Brigitte Maurice-Chabard, Paris, Picard, 1999, p. 169-191.

Jung, Marc-René, "Les "Douze Dames de rhétorique” ", in Du mot au texte. Actes du III colloque international sur le moyen français, éd. par Peter Wunderli, Tübingen, Narr, 1982, p. 229-240 (Tübinger Beiträge zur Linguistik, 175).

Lapaire, Claude, "Les stalles de la chapelle des Martyrs thébains", in La cathédrale de Lausanne, éd. par Jean-Charles Biaudet et al., Berne, Société d'histoire de l'art en Suisse, 1975, p. 214-220.

—, "Les stalles de la cathédrale de Lausanne», in Stalles de la Savoie médiévale, catalogue de l'exposition, Genève, Musée d'art et d'histoire de Genève, 1991, p. 201-206.

Montfalcon, Louis de, "Aymon de Montfalcon, prélat de la Renaissance», Le Bugey, 55 (1968), p. 73-109; 56 (1969), p. 178$200 ; 57$ (1970), p. 149-172.

Mühlethaler, Jean-Claude, "Quand Fortune, ce sont les hommes. Aspects de la démythification de la déesse, d'Adam de la Halle à Alain Chartier", in La Fortune. Thèmes, représentations, discours, éd. par Yasmina Foehr-Janssens, Emmanuelle Métry, Genève, Droz, 2003, p. 177-206. 
—, «Ein dichterisches und politisches Manifest in Lausanne. Zur Interpretation des Breviaire des Nobles und der Douze Dames de Rhétorique in den Fresken des Schlosses Saint-Maire aus literaturhistorischer Sicht", in Paroles de Murs/Sprechende Wände, dir. par Eckart Conrad Lutz, Dominique Rigaux, Grenoble, Cahiers du CRHIPA 10, 2007, p. 91-105.

—, "Satire et recyclage littéraire: la fureur de Mégère, de Regnaud Le Queux à Antitus Favre", in La question du sens au Moyen Âge. Hommage au professeur Armand Strubel, éd. par Dominique Boutet, Catherine Nicolas, Paris, Champion, 2017, p. 605-623.

Piaget, Arthur, "Aymon de Montfaucon et sa cour littéraire", Pages d'histoire neuchâteloise, 45 (1935), p. 169-189.

Pradervand, Brigitte, "Les peintures murales du château Saint-Maire», in Le château Saint-Maire XIV $V^{e} X X I^{e}$ siècle, Canton de Vaud, CADEV, 2018, p. 68-83.

Pradervand, Brigitte, Schätti, Nicolas, «Les stalles», in La cathédrale Notre-Dame de Lausanne. Monument européen, temple vaudois, éd. par Peter Kurmann, Lausanne, La Bibliothèque des arts, 2012, p. 183-191.

Reymond, Maxime, «Si qua fata sinant», Les Échos de Saint-Maurice, 30 (1931), p. 21-23.

—, «Un peintre dijonnais à Lausanne au $\mathrm{XV}^{\mathrm{e}}$ siècle», Feuille d'avis de Lausanne, 2 avril 1938.

Stauffer, Annemarie, D'or et de soie ou les voies du salut. Les ornements sacerdotaux d'Aymon de Montfalcon, évêque de Lausanne, catalogue d'exposition, Berne, Musée d'histoire, 2001.

Straub, Karen, Les Douze Dames de Rhétorique, Studien zu einer französischen Handschrift des 15. Jahrhunderts, Dissertation, Berlin, Institut für Kunstwissenschaft und Historische Urbanistik, 2011. 
